\title{
Skén\&graphie
}

SKIEN\&GAPHIIU Coulisses des arts du spectacle et des scènes

émergentes

$6 \mid 2019$

Voyages des cultures et des mémoires dans les arts de la scène

\section{Antigone comme symbole de la lutte en faveur de toutes et tous les « sans deuil »: Darja Stocker sur la notion de "génération » et l'héritage de 1968}

Entretien avec Darja Stocker

Catherine Mazellier-Lajarrige

\section{OpenEdition \\ Journals}

Édition électronique

URL : https://journals.openedition.org/skenegraphie/3065

DOI : 10.4000/skenegraphie.3065

ISSN : 2553-1875

\section{Éditeur}

Presses universitaires de Franche-Comté

\section{Édition imprimée}

Date de publication : 1 novembre 2019

Pagination : 209-213

ISBN : 978-2-84867-673-9

ISSN : $1150-594 X$

\section{Référence électronique}

Catherine Mazellier-Lajarrige, «Antigone comme symbole de la lutte en faveur de toutes et tous les « sans deuil » : Darja Stocker sur la notion de « génération » et l'héritage de 1968 », Skén\&graphie [En ligne], 6 | 2019, mis en ligne le 01 juin 2021, consulté le 27 septembre 2021. URL : http://

journals.openedition.org/skenegraphie/3065; DOI : https://doi.org/10.4000/skenegraphie.3065

Ce document a été généré automatiquement le 27 septembre 2021.

Presses universitaires de Franche-Comté 


\section{Antigone comme symbole de la lutte en faveur de toutes et tous les « sans deuil » : Darja Stocker sur la notion de " génération » et l'héritage de 1968}

Entretien avec Darja Stocker

Catherine Mazellier-Lajarrige

\section{NOTE DE L'ÉDITEUR}

Propos recueillis et traduits par Catherine Mazellier-Lajarrige.

Catherine Mazellier-Lajarrige : La thématique générationnelle est-elle fructueuse ou pertinente pour toi en tant que dramaturge?

Effectivement, cette thématique s'est de plus en plus imposée à moi ces dernières années. Dans ma dernière pièce, Nulle part en paix. Antigone, une adaptation de la version de Sophocle, j'ai pris le parti de n'avoir pas une seule, mais trois Antigones dans la pièce. Et j'aurais même pu en avoir 5,7, voire plus de 20, car Antigone est davantage qu'un personnage, elle symbolise la lutte en faveur de toutes et tous les «sans deuil ", comme les appelle Butler; une lutte qui rend sensible au fait qu'il existe des personnes dont le deuil, ou la mort, n'importe pas.

Dans ma pièce, Antigone devient le projet d'une génération, qui ne rassemble pas seulement des membres de cette génération, mais toutes celles et tous ceux qui se reconnaissent en lui (par exemple, le jeune Hémon et le garde plus âgé rejoignent le mouvement vers la fin de la pièce). La notion de « génération » devient pertinente pour moi à partir du moment où je remarque que je ne peux plus parler uniquement pour moi, en tant qu'individu. Mon expérience est certes singulière, elle est le fruit des 
influences de la société sur moi en tant que femme blanche, née avec un passeport suisse et un passeport européen, issue d'une famille peu nantie etc., mais je ne suis pas non plus exclusivement influencée par ma position dans la société, mais aussi par ce que d'autres, qui ont grandi autour de moi dans d'autres conditions, vivent avec moi et à côté de moi.

4 Je ne peux certes pas prétendre parler pour d'autres que moi, mais lorsque j'écris, il m'est impossible de me percevoir comme isolée: j'appartiens à une génération caractérisée par la diversité des perspectives, à un point inconnu jusqu'alors. C'est cette constante multiplicité des expériences, passées ou présentes, qui la caractérise selon moi. Cet éclatement la fragmente, mais il est aussi ce qui la relie. Aujourd'hui, dans mon adaptation d'Antigone, je ne peux plus parler d'un cas individuel, d'une héroïne, d'une femme courageuse en particulier : je ne peux que parler de ma génération, qui ne veut plus accepter l'état du monde tel qu'il est. Je ne peux pas non plus ignorer que les choses ont changé depuis Sophocle, voire Brecht: les femmes ne sont plus seules dans leur lutte. Je ne pouvais donc pas laisser Antigone toute seule. Dans ma pièce, les Antigones sont issus de différents milieux sociaux : l'une d'elle, la plus privilégiée, est journaliste, elle voyage bien au-delà des frontières de l'Europe et devient témoin d'un régime répressif, l'autre vient du Sud de l'Europe et tente d'agir contre la politique européenne de contrôle des frontières, la troisième est assistante sociale dans un quartier défavorisé d'une métropole/grande ville européenne. Cette diffraction d'Antigone en différentes personnalités engagées dans différents lieux géographiques et pensant/conduites à penser leurs privilèges amorce une réflexion sur l'extrême diversité de ma génération, d'un part, mais aussi sur les liens qui la tissent. Dans mon interprétation, toutes les Antigones ont grandi en Europe, mais en dépit de cette focalisation, il y a des possibilités innombrables de parcours de vie. À cela s'ajoute qu'elles sont reliées à d'autres membres de la même génération par-delà les frontières de l'Europe. En tant qu'autrice, il m'est impossible de ne pas me préoccuper des jeunes gens de mon âge, hommes et femmes, qui tiennent bon dans un hôpital en Syrie pour opérer des blessés, se rendent dans des zones à risque pour leur activité de journalistes, croupissent en prison, sont obligés de fuir ou de vivre en exil. Avec les mouvements actuels de fuite ou migration et leur présence dans les médias sociaux, on ne peut plus détourner le regard. Même les responsables des attentats à Paris ou à Bruxelles avaient mon âge, ils ont grandi dans la même Europe que moi, et lorsque j'écris, je suis forcée de me demander pourquoi ils n'ont pas eu de voix, pourquoi ils n'ont pas eu d'autre langage que celui de la violence explosive et de l'autodestruction. La notion de "génération" n'est pas un sujet sur lequel je choisirais d'écrire, comme on peut étudier je ne sais quel phénomène de la culture jeune. Pour moi, elle signifie plutôt que je me sens reliée aux personnes de mon âge en divers lieux, indépendamment de la distance géographique ou du milieu d'origine.

Quel rôle ont joué pour toi les idéaux de la génération de 1968 ? L'influence de cette génération est-elle perceptible aujourd'hui encore?

61968 a permis la conquête de certaines choses que je considère aujourd'hui comme allant de soi. Le fait, par exemple, que je puisse décider moi-même, en tant que femme, de mon parcours professionnel. Ou que je puisse avoir mon propre compte bancaire. Même si les structures sont restées patriarcales et que s'y sont ajoutés des mécanismes de pouvoir néolibéraux, je peux au moins tabler sur le fait que nul ne me refuse le droit à vouloir déterminer moi-même ma trajectoire. Dans le domaine du théâtre, que ce soit 
à l'Université ou dans mon quotidien professionnel, les soixante-huitards que j'ai rencontrés m'ont semblé incarner davantage la survivance de structures patriarcales que la défense de leurs idéaux.

7 Si l'on manifestait soi-même un engagement en tant que femme, voire jeune femme, que l'on voulait élargir l'horizon à d'autres territoires, c'était perçu comme une menace. Si l'on remettait en cause la position de pouvoir du professeur, du directeur de théâtre, etc., beaucoup plus âgé, l'engagement de l'époque était utilisé comme argument pour dire qu'on était trop jeune pour savoir. Sur le plan politique, j'ai souvent été confrontée à des partis-pris idéologiques, et en matière de relations homme-femme, à une attitude patriarcale de monarque.

Pour certains soixante-huitards, le fait que ma génération perçoive le monde de manière différenciée, dans sa complexité et ses contradictions, semblait presque remettre en cause leur identité. Même chose pour la disparition de la frontière entre « nous » et « les autres », c'est-à-dire le fait qu'on ne puisse plus dire clairement qui est "nous » et qui sont «les autres", parce que les familles et les amis sont éparpillés à travers les continents: cela semblait détruire leur schéma fondamental de l'aide solidaire, leur image de l'activiste politique qui s'investit pour défendre les droits des autres. En résumé, on pourrait dire que j'ai été très influencée par les idéaux et l'engagement, mais que j'ai été jusqu'à présent déçue par la relation directe avec des représentants, parfois autoproclamés, de ce mouvement, surtout des hommes. À cela s'ajoute le fait que mes parents appartiennent à la génération post-68, ils avaient environ 20 ans dans les années 80 et étaient déjà confrontés eux-mêmes à l'héritage de 1968. Les mouvements en France et en Allemagne ont été liés, et pourtant, en parlant aux uns et aux autres, j'ai eu l'impression que cela faisait une grande différence d'avoir connu l'engagement dans l'Allemagne divisé et de l'avoir vécu en France. Comme ma famille est originaire de Suisse et de France, j'ai été "dès le départ», davantage influencée par les années 60 dans ces deux pays, ou en tout cas par la prise de position de mes parents par rapport à ce contexte. C'est seulement à partir de vingt ans, quand je me suis installée à Berlin, que je suis entrée en contact avec des représentants de 1968 en Allemagne. Autant que je puisse en juger, ce sont les mouvements des années 80 et 90 qui ont été précurseurs des mouvements actuels : ce n'étaient plus seulement des révoltes étudiantes, mais des initiatives citoyennes rassemblant plusieurs couches sociales. Il serait intéressant d'étudier dans quelle mesure elles ont été présentées comme moins marquantes parce qu'elles n'avaient pas de leaders universitaires et que beaucoup de leurs « enfants » n'avaient pas vraiment de lobby pour immortaliser dans l'opinion publique leurs récits et leurs luttes. En tout cas, on a déjà pu voir se dessiner dans ces initiatives les contradictions d'un combat dépassant les frontières des origines sociales ou ethniques. À Berlin comme à Zurich, les structures qui sont nées dans les années 1980-90 sont à présent des lieux où les réfugié-e-s peuvent trouver un hébergement et des repères. 


\section{AUTEUR}

\section{CATHERINE MAZELLIER-LAJARRIGE}

Maîtresse de conférences en Études Germaniques à l'Université Toulouse-Jean Jaurès (UT2J) et membre du CREG (Centre de Recherches et d'Études Germaniques, http://creg.univ-tlse2.fr). Ses recherches et publications portent sur le théâtre des $\mathrm{XX}^{\mathrm{e}}$ et $\mathrm{XXI}^{\mathrm{e}}$ siècles, la pantomime, ainsi que les phénomènes de sécularisation dans la littérature germanophone. Elle dirige aux Presses Universitaires du Midi les collections de théâtre contemporain étranger « nouvelles scènes » et en codirige la déclinaison pour le théâtre germanophone. 